\title{
Recombinant Newcastle disease virus expressing P53 demonstrates promising antitumor efficiency in hepatoma model
}

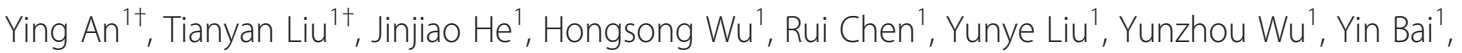
Xiaochen Guo', Qi Zheng ${ }^{1}$, Chang Liu', Jiechao Yin', Deshan Li ${ }^{1}{ }^{1} 2^{*}$ and Guiping Ren ${ }^{1,2^{*}}$

\begin{abstract}
Background: Numerous studies have demonstrated that the NDV-mediated gene therapy is a promising new approach for treatment of cancers. P53 plays a vital role in tumor suppression and surveillance. Therefore, we hypothesize that a recombinant NDV expressing P53 would be an ideal agent for the hepatoma therapy.

Results: In the essay, the human P53 gene was incorporated into the genome of a lentogenic strain (named rNDV-P53), which did not affect viral replication kinetics and magnitude in HepG2 cells. Compared to the vehicle virus, rNDV-P53 increased cell growth suppressor ratio and early apoptosis by 2 folds, and decreased the mitochondrial membrane potential in HepG2 cells. In vivo studies, treatment with rNDV-P53 reduced tumor volume of tumor-bearing mice by more than 4 folds, tumor weight by more than 5 folds comparing with rNDV. The 120-day survival rate of rNDV-P53-treated mice was $75 \%$, survival rate of rNDV-treated mice was $12.5 \%$. TUNEL analysis showed a significant increase in the apoptosis rate in the tumor tissues of rNDV-P53-treated mice than that of rNDV-treated mice. Moreover, serum chemistries revealed an insignificant change of blood urea nitrogen (BUN), creatinine levels, alanine aminotransferase (ALT) and aspartate transaminase (AST) in rNDV-P53-treated group compared to normal mice, suggesting treatment with the recombinant virus was not toxic.
\end{abstract}

Conclusion: rNDV-P53 is a potent candidate for carcinoma therapy especially for hepatocarcinoma.

Keywords: Recombinant NDV, P53, Gene therapy, Anti-tumor

\section{Background}

Newcastle disease virus (NDV) is a single-strand nonsegmented negative-sense RNA virus of the paramyxoviridae family [1]. Causing severe infection to multiple avian species, but is not pathogenic to humans. The genome containing 15186 nucleotides encodes six genes, including the nucleocapsid protein $(\mathrm{NP})$, phosphoprotein $(\mathrm{P})$, matrix protein $(\mathrm{M})$, fusion protein $(\mathrm{F})$, hemagglutinin neuraminidase $(\mathrm{HN})$ and RNA-dependent RNA polymerase (L) [2, 3]. NDV was proposed as a promising anticancer agent [4-7], it is prone to replicate in human tumor cells and cause oncolytic effects but not in normal cells [8-10],

\footnotetext{
*Correspondence: deshanli@163.com; renguiping@126.com

${ }^{\dagger}$ Equal contributors

${ }^{1}$ Biopharmaceutical Lab, College of Life Science, Northeast Agriculture University, Mucai Street 59, Xiangfang district, Harbin, People's Republic of China

Full list of author information is available at the end of the article
}

which is a superior characteristic as a therapeutic agent for cancers compared with the other oncolytic virus. The anticancer potency of NDV depends on the ability to induce apoptosis in infected cancer cells [11-13]. However, previous studies have demonstrated that NDV induces apoptosis in tumor cells independent of the function of the P53 $[14,15]$.

The P53 tumor suppressor plays various roles in cellcycle control, apoptosis, senescence, DNA repair and modulating metabolic processes [16-20]. As a transcription factor, P53 can regulate several proteins, such as pro-apoptotic Bcl-2, caspases, death receptors and so on. With the expression of these genes, cell death was induced by apoptosis [21, 22]. Meanwhile, the tumor suppressor P53 is inactivated by mutations, which can be found in about $50 \%$ of human cancers [23, 24]. It has been proposed that the increased survival of tumor cells 
lacking functional P53 is due to the decreased ability of these cells to undergo apoptosis [25]. Briefly, the expression of wt-P53 gene can inhibit tumorigenesis. It is not surprising that restoring wild-type P53 activity has been used as a cancer gene therapy in both laboratory studies and clinical trials [26-29].

In the present study, we generated a recombinant NDV (named rNDV-P53) and evaluated its antitumor efficacy in vitro and in vivo tests. We showed that the virus possesses a significant oncolytic activity against hepatoma cell line HepG2 and is an effective oncolytic agent in a H22 tumor mouse model. In a conclusion, we conclude that recombinant NDV expressing P53 is a promising agent for cancer therapy.

\section{Methods}

\section{Cell culture}

The human hepatoma cell line of HepG2, Hep3B and the mice hepatoma cell line of $\mathrm{H} 22$ were supplied by northeast agricultural university biological pharmaceutical teaching and research section. The baby hamster kidney cell line of BHK21 was a generous gift from Dr. B.Moss. HepG2 and BHK21 cells were cultured in DMEM containing $10 \%$ new-born calf serum (NCS) and $1 \%$ penicillin/streptomycin. All cell lines were maintained at $37{ }^{\circ} \mathrm{C}$ in a $5 \% \mathrm{CO}_{2}$ atmosphere and $95 \%$ humidity.

\section{Recombinant Newcastle disease virus}

The total RNA of human peripheral blood leukocytes was prepared by using Trizol and then was transcribed into cDNA. The P53 gene was amplified by PCR using the human peripheral blood leukocytes cDNA as template and the following primers: sense 5'-ATGGAG GAGCCGCAG-3' and antisense 5'-TCAGTCTGAGTC AGGCCCTT-3'. The PCR product was purified by $1 \%$ agarose gel electrophoresis and inserted into HpaI-MluI fragment of clone 30 plasmid. The nucleotide sequence was identified by sequence analysis and compared with the reported P53 gene [GenBank: 82395019]. And then the recombinant plasmid was transiently cotransfected with helper plasmids encoding viral NP, P and L into BHK21 cells stably expressing T7 RNA polymerase using lipofectamine 2000. The virus was rescued and amplified by inoculation of the supernatant from the transfected cells into the allantoic cavity of specific-pathogen free chicken embryos.

\section{Determination of virus growth}

Virus growth was determined in HepG2 cells culture. HepG2 cells in 6-well plates were infected with recombinant virus at $37{ }^{\circ} \mathrm{C}$ in DMEM supplemented with $10 \%$ new-born calf serum and $1 \%$ penicillin/streptomycin in a $5 \% \mathrm{CO}_{2}$ atmosphere. Cells with supernatants were frozen at the time indicated i.e. 24, 48, $72,96 \mathrm{~h}$ postinfection. After repeated freezing and thawing 3 times, the cells with supernatants were collected. The concentration of virus was determined by end-point titration on chicken embryo fibroblast cells and was expressed as mean $\log _{10} 50 \%$ tissue culture infective dose $\left(\mathrm{TCID}_{50}\right)$ per ml. Finally, according to the virus titer in different time a growth curve was drawn.

\section{Determination of exogenous P53 protein expression by Western blotting}

HepG2 and Hep3B cells $\left(5 \times 10^{6}\right.$ cells $)$ were infected with rNDV-P53 at 1 MOI. After $24 \mathrm{~h}$ incubation, cells were collected and washed twice with cold PBS by centrifugation at $500 \times \mathrm{g}$ for $5 \mathrm{~min}$ at $4{ }^{\circ} \mathrm{C}$. The pellet was resuspended in lysis buffer supplemented with proteases inhibitor and the supernatant was stored at $-20{ }^{\circ} \mathrm{C}$. For western blotting analysis, samples were separated by $10 \%$ sodium dodecylsulfate-poly acrylamide gel electrophoresis (SDS-PAGE), and transferred to a nitrocellulose membrane. The blot was visualized by chemiluminescence and autoradiography using X-ray film. Mouse anti- human P53 polyclonal antibody (DO-1) was obtained from Santa Cruz Biotechnology Inc., CA, USA. A protein marker (New England Biolabs, Beverly, MA, USA) was run for each gel to identify P53.

\section{Cell viability assay}

A short-term microculture tetrazolium (MTT) assay was used to quantify cell viability. Approximately $2 \times 10^{4}$ HepG2 cells were plated into 96-well plates in complete medium and allowed to attach for $24 \mathrm{~h}$. Subsequently, the cells infected with rNDV-P53, rNDV at $0.01 \mathrm{MOI}$, $0.1 \mathrm{MOI}, 1 \mathrm{MOI}$, and $10 \mathrm{MOI}$ in triplicate. After $48 \mathrm{~h}$ of incubation, $20 \mu \mathrm{l}$ MTT solutions $(5 \mathrm{mg} / \mathrm{ml}$ in sterile phosphate-buffered saline) were added to the cell. $4 \mathrm{~h}$ later, the MTT solution in the wells was discarded, then $150 \mu \mathrm{l}$ dimethyl sulfoxide (DMSO) was added. The absorption at $490 \mathrm{~nm}$ (OD490) was measured on a microplate reader. The cell viability was converted and expressed as the percentage of the control. The cells without any treatment were used as negative control.

Inhibition rate $\%=($ control group OD - treatment group OD) $/$ control group OD $\times 100 \%$

\section{Annexin V -FITC and PI binding assay}

Approximately $2 \times 10^{4}$ HepG2 cells were plated into 6well plates in complete medium and allowed to attach for $24 \mathrm{~h}$. Subsequently, the cell infected with rNDV-P53, rNDV at $0.1 \mathrm{MOI}, 1 \mathrm{MOI}$, and $10 \mathrm{MOI}$ in triplicate. After $48 \mathrm{~h}$ of incubation, cells $\left(0.5 \sim 1 \times 10^{6}\right)$ were digested with $0.25 \%$ trypsin, then resuspended by 
adding $200 \mu \mathrm{l}$ Binding Buffer. Then the cells were incubated away from light with $5 \mu \mathrm{l}$ Annexin V labelled FITC $30 \mathrm{~min}$ at room temperature. At last, after being mixed with $5 \mu \mathrm{l}$ PI and $300 \mu \mathrm{l}$ Binding Buffer and reacted $5 \sim 15$ min away from light, the cells were quantitatively detected by flow cytometry (usually within $1 \mathrm{~h}$ ), at the same time a tube cells without AnnexinV-FITC and PI served as a negative control. Cells were analyzed in a flow cytometry using FL1 $(530 \mathrm{~nm})$ bandpass filters of FACS Calibur (Bectom Dickinson, USA), and data were analyzed by the CellQuest software (BD, USA).

\section{Measurement of mitochondrial membrane potential}

Changes in mitochondrial transmembrane potential $(\triangle \Psi M)$ were measured by using $5,5^{\prime}, 6,6^{\prime}$-tetrachloro1,1',3,3' 'tetra-ethylbenzimidazolylcarbocyanine iodide (JC-1) as a probe. HepG2 cells were plated in six-well plates at $1 \times 10^{5}$ cells/well and infected by the recombinant virus rNDV-P53 and rNDV at 1 MOI. After 12 or $24 \mathrm{~h}$ of incubation, cells were digested with $0.25 \%$ trypsin, then resuspended in $1 \mathrm{ml}$ DMEM containing JC-1 $(20 \mu \mathrm{g} / \mathrm{ml})$. Then cells were incubated for $30 \mathrm{~min}$ in dark at room temperature. Cells were washed twice with PBS, re-suspended in $0.5 \mathrm{ml} \mathrm{PBS}$, and immediately analyzed in a flow cytometry using FL1 $(590 \mathrm{~nm})$ band pass filters of FACS Calibur (Bectom Dickinson, USA).

\section{In vivo evaluation of tumor growth}

All procedures involving animals followed the guidelines issued by National Institute of Health and the Institutional Animal Care and Use Committee of Northeast Agriculture University (approval number: SCXK-2011-0004).

Six-week-old male ICR mice (with a mean weight of $18 \mathrm{~g}$ ) were housed in a pathogen-free environment and implanted subcutaneously with $2 \times 106$ H22 cells into the right groin. When the tumor size reached about $50 \mathrm{~mm} 3$, the animals were randomly divided into three groups. Then the tumor-bearing mice were intratumoral injection inoculated with a single injection of $1 \times 107 \mathrm{pfu}$ of rNDV-P53 or rNDV in a $200 \mu \mathrm{l}$ volume and $200 \mu \mathrm{l}$ of PBS were injected instead of the virus, which represented as control. Tumor growth was observed every 2 days for 18 days by measuring the two dimensional longest axis (L) and shortest axis (W) with a caliper. The tumor volume was calculated by using the following formula: volume in $\mathrm{mm} 3=4 / 3 \times \pi \times(\mathrm{L} / 2 \times \mathrm{W} / 2 \times \mathrm{W} / 2)$. According to the institutional protocols, the mice were euthanized when the tumors reached $18 \mathrm{~mm}$ in diameter. On day 18, all mice from the control group and 6 mice from each treatment group were sacrificed and their tumors were excised. The tumors were weighted and immersed in $4 \%$ paraformaldehyde. Then all sections were cut at $4 \mathrm{~mm}$ thick from buffered formalin- fixed, paraffin-embedded tumors. After deparaffinization, sections were stained with haematoxylin/eosin (H\&E) and prepared for TUNEL assay (terminal deosynucleotidy transferase-mediated dUTP nick and labeling assay). In addition, serum was prepared from whole blood for determination of serum concentrations of AST, ALT, BUN, and creatinine. The remaining mice in each treatment group were observed for 120 days with measurement of tumor volume. This was disease-free 120-day survival rate.

\section{Statistical analysis}

All the data were obtained from at least three independent experiments. The data are expressed as mean values \pm SD and were compared using Student's t test. Statistical significance was defined as a $\mathrm{P}$ value $<0.05$.

\section{Results}

\section{Recovery of the recombinant virus rNDV-P53}

The human wild-type P53 gene was inserted into the newly created Hpa I and Mlu I sites between the F and HN genes of the NDV genome (Fig. 1). Then we generated the recombinant virus rNDV-P53. The incorporated plasmid was transfected into BHK-21 cells. Then the supernatant of the transfected monolayers was injected into 10-day-old SPF embryonated chicken eggs. After 3 days, the allantoic fluid was harvested and analyzed in a HA test. The high titers of rNDV-P53 suggested the successful generation of the recombinant NDV virus.

\section{Growth and exogenous P53 protein expression of the recombinant virus rNDV-P53}

In order to verify the recombinant virus rNDV-P53 remains stable proliferation in tumor cells, HepG2 cells were infected with rNDV-P53 at MOI of 1 and the supernatant of them was harvested at different time points. Then the viral titers in the supernatants were determined in triplicate. As shown in Fig. 2a, compared with the parental virus, the recombinant virus rNDVP53 has no any distinctions in the kinetics and magnitude of replication while the production of rNDV-P53 was slightly delayed. To investigate the expression of

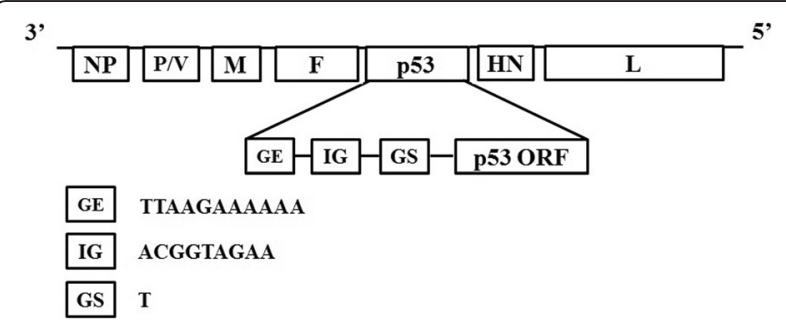

Fig. 1 Construction of rNDV-P53. Diagram showing insertion of the P53 gene into the NDV genome at the position between the $\mathrm{F}$ and $\mathrm{HN}$ genes 


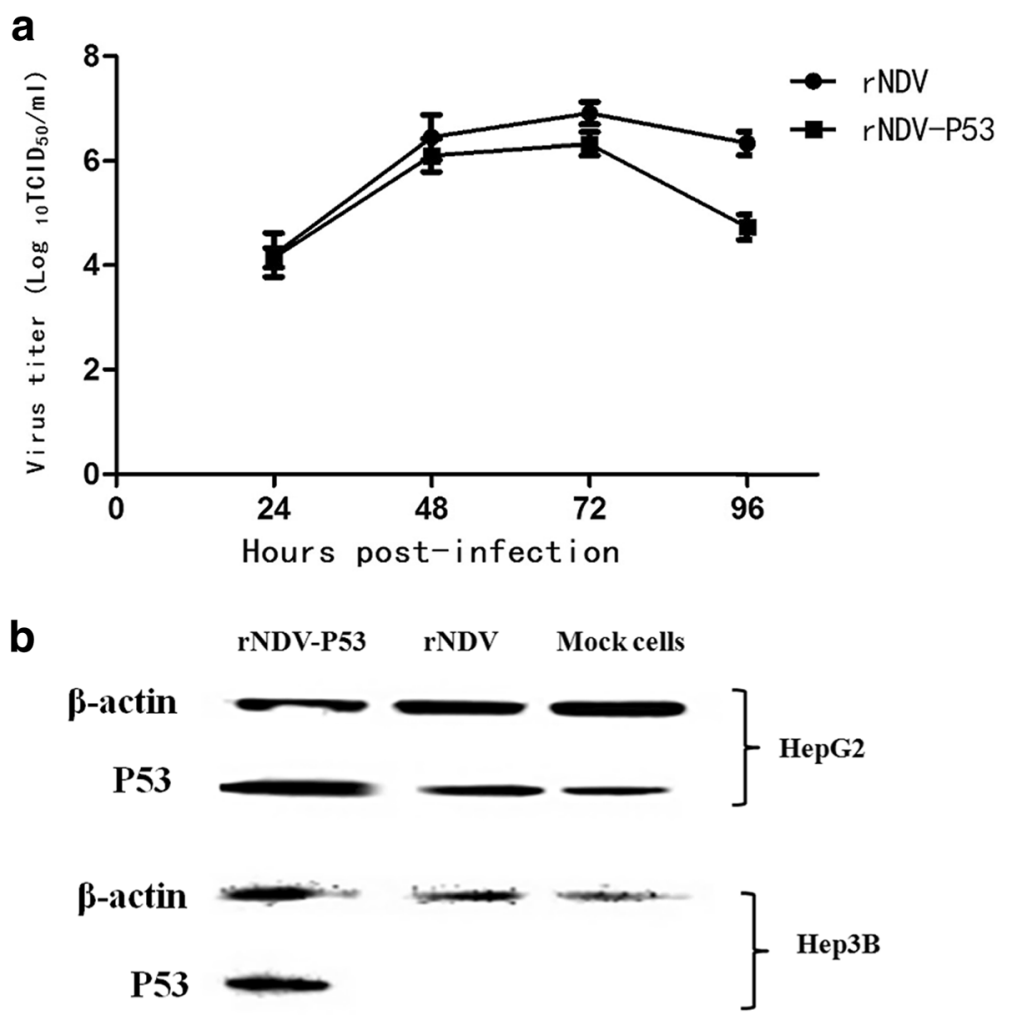

Fig. 2 Growth curve of the recombinant virus and Exogenous P53 protein expression. a The virus titers in the supernatant of different time points were assessed by plating tenfold dilution on DF-1 cells and were shown as $\log _{10} T C I D_{50} / \mathrm{ml}$. $\mathbf{b}$ The $\beta$-actin was used as control

P53 protein, HepG2 and Hep3B cells were treated with rNDV-P53 at MOI of 1 . Western blotting analysis (Fig. 2b) showed that the P53 expression increased in the tumor cells infected with rNDV-P53.

\section{Recombinant virus rNDV-P53 suppresses tumor cell growth in vitro}

The direct cytotoxic activity of the recombinant virus rNDV-P53 was tested in HepG2 tumor cells in this study. As shown in Fig. 3a, rNDV-P53 induced cell death in a dose-dependent manner during 24 hour's treatment in HepG2 cells. When the cells were infected with rNDV-P53 or rNDV of $10 \mathrm{MOI}$ for $24 \mathrm{~h}$, the viability of HepG2 cells dramatically decreased to $45.03 \% \pm 3.6 \%$, $73.67 \% \pm 2.51 \%$, respectively. In the meanwhile, the cell growth suppressor ratio induced by rNDV-P53 is up to 1.7 times of $\operatorname{rNDV}(\mathrm{MOI}=1)$. Collectively, these data demonstrate that the recombinant virus rNDV-P53 is superior to parent virus in suppressing the growth of HepG2 cells.

To determine the sensitivity of tumor cell to recombinant virus-induced apoptosis, HepG2 cells were treated with recombinant virus rNDV-P53 for $24 \mathrm{~h}$, and the percent of apoptotic cells was determined by Annexin V-FITC/PI assay. Apoptosis assay showed that after virus infection the tumor cell death by apoptosis was occurred markedly. The results showed that apoptosis percentages of rNDV group and rNDV-P53 were $10.4 \%$ and $21.1 \%$ respectively, which indicated that virus rNDV-P53 were more effective in inducing apoptosis in the HepG2 cells (Fig. 3b). In line with the observation of cell death, rNDV-P53 induced apoptosis in a dose-dependent manner.

To explore the molecular mechanisms by which virus induced cell death, the mitochondrial membrane potential of HepG2 cells infected with virus was assayed by using the fluorescent dye JC-1. JC-1 accumulates in mitochondria as aggregates, resulting in red fluorescence in non-apoptotic cells. The monomeric form, which fluoresces green, is found in apoptotic cells. As shown in Fig. 3c, the green fluorescence of JC-1 increased in HepG2 cells treated with virus over the time course. Both rNDV and rNDVP53 reduced the mitochondrial membrane potential compared with the PBS group, but the effect of rNDV was not as potent as rNDV-P53. These results indicate that the recombinant virus can effectively reduce the mitochondrial membrane potential, which is the key in cell apoptosis.

These data suggest that the insertion of P53 gene improved the ability of NDV inducing tumor cell apoptosis. 

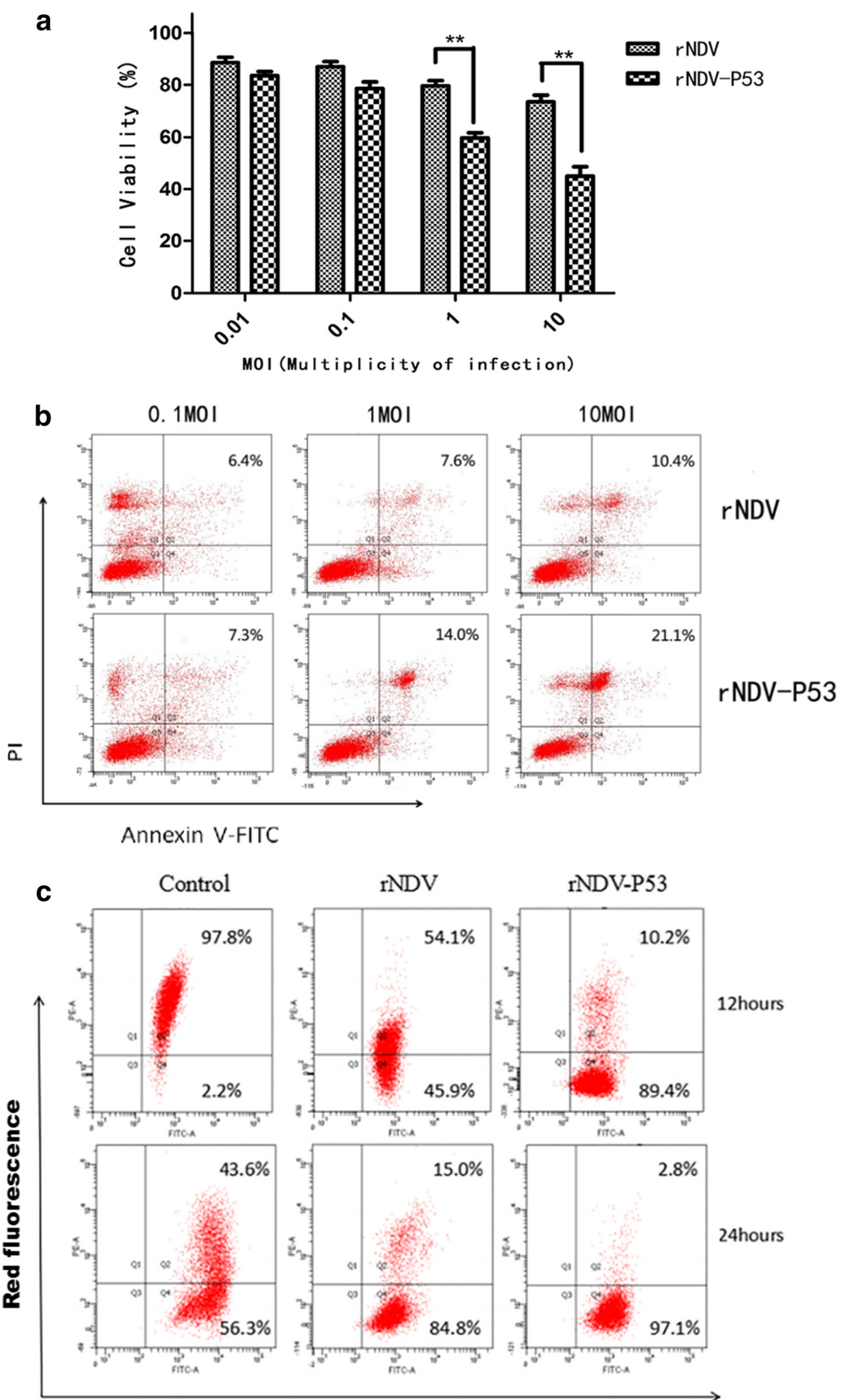

Green fluorescence

Fig. 3 (See legend on next page.) 
(See figure on previous page.)

Fig. 3 The recombinant virus rNDV-P53 induces cell death by apoptosis in human tumor cells HepG2. a Cytotoxicity of rNDV-P53 in tumor cells. HepG2 cell were infected with rNDV or rNDV-P53 virus for $24 \mathrm{~h}$. Cell viability was determined by MTT assays (mean \pm SD). Replicates were averaged, and the error bars represent the SD of three independent experiments. $\mathbf{b}$ The cells were infected with the rNDV or rNDV-P53 in different dosages. Cell apoptosis was detected by Annexin V-FITC/PI Apoptosis Detection Kit according to the manufacturer's instructions and followed by flow cytometry. c After $12 \mathrm{~h}$ or $24 \mathrm{~h}$ of virus infection, HepG2 cells were stained with JC-1 and analyzed by flow cytometry

\section{The recombinant virus rNDV-P53 suppresses tumor} growth in mice

The mice $\mathrm{H} 22$ hepatocellular carcinoma model was employed in our study for examining the tumor suppressing efficacy of the recombinant virus rNDV-P53 in vivo. When the tumor size reached about $50 \mathrm{~mm}^{3}$, the animals were divided into three groups. The mice were intratumorally treated with $1 \times 10^{7}$ pfu of rNDV, rNDVP53 or PBS every other day for nine injections. The tumor growth was observed by measuring the tumor size every other day for a total of 18 days. After nine injections, all animals in the PBS group were sacrificed

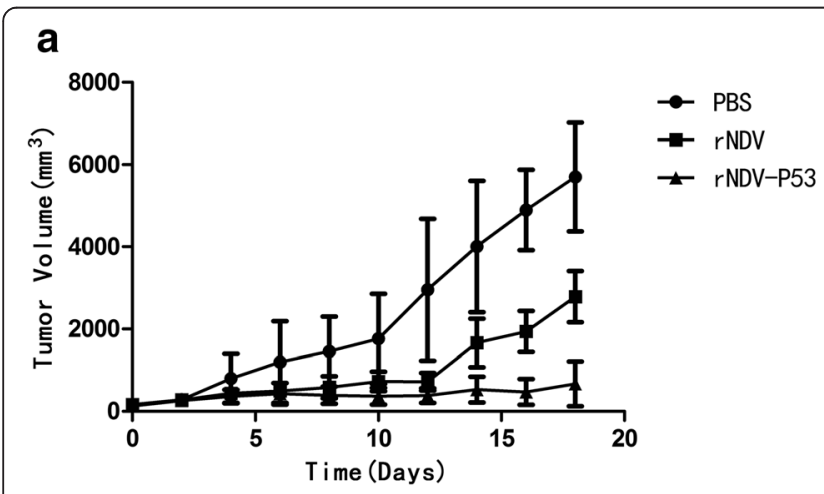

C

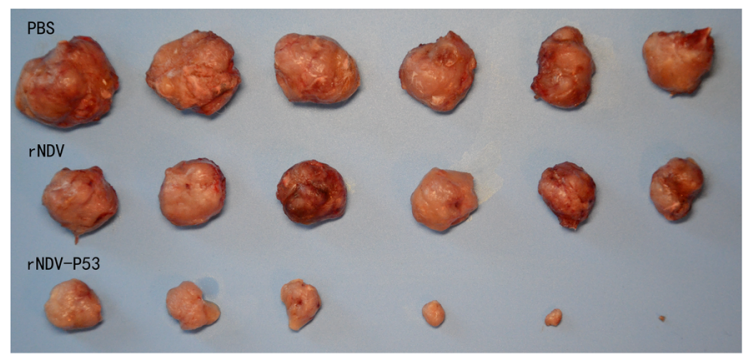

b

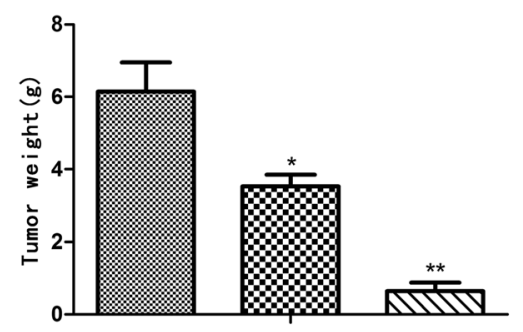

d

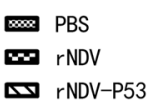

PBS

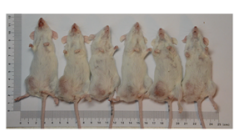

rNDV

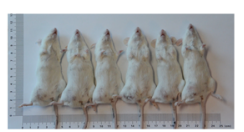

rNDV-P53

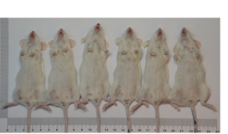

e

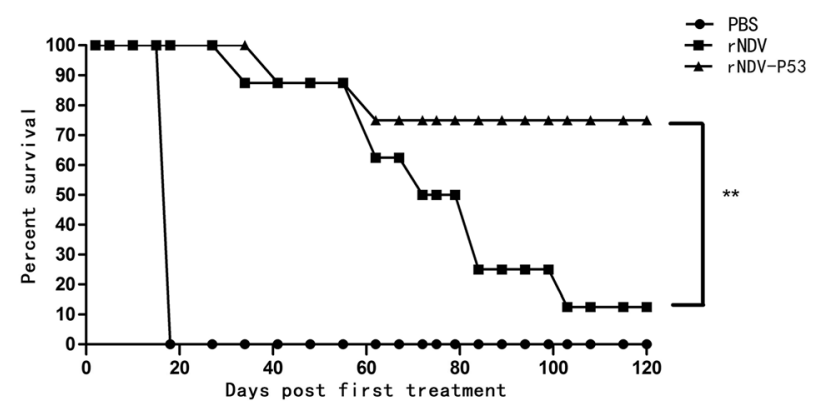

Fig. 4 The recombinant virus rNDV-P53 suppresses the growth of H22 xenografts in mice. A xenograft H22 animal model was established in mice. When the tumor size reached to about $50 \mathrm{~mm}^{3}$ (about 7 days post-inoculation), the tumors were injected with $1 \times 10^{7}$ pfu of rNDV or rNDV-P53 virus (day 0). The PBS was used as control. The injections were repeated every 2 days during experimental period. Mice were killed when the tumors reached $18 \mathrm{~mm}$ in length. On day 18, all mice from the control group and 6 mice from each treatment group were sacrificed and their tumors were excised. The remaining 8 mice in each treatment group were observed for 120 days with measurement of tumor volume. a The tumor growth was observed by measuring the tumor size every 2 days for 18 days. $\mathbf{b}$ The weight of the excised tumors of different groups. $\mathbf{c}$ The excised tumors of different groups. $\mathbf{d}$ Before necropsy, the tumor in the right groin of different groups mice. e The percent survival of tumor-bearing mice treated with PBS, rNDV, and rNDV-P53 in the 120 days $\left({ }^{* *} p<0.01 ;{ }^{*} p<0.05\right)$ 
due to excessive tumor burden. The average tumor size for the PBS-control group was $5702.93 \pm 540.25 \mathrm{~mm}^{3}$, while $666.96 \pm 222.62 \mathrm{~mm}^{3}$ noted for the rNDV-P53 group. For the group treated with rNDV, the average tumor sizes were $2785.45 \pm 254.30 \mathrm{~mm}^{3}$ (Fig. 4a). Moreover, the average tumor weight of PBS-control group was $6.14 \pm 0.81 \mathrm{~g}$. For the group treated with rNDV and rNDV-P53, the average tumor weight was $3.52 \pm 0.33 \mathrm{~g}$ and $0.64 \pm 0.24$ g, respectively (Fig. 4b). During necropsy, tumors excised from the control group were fairly large in size and tumors isolated from the rNDV-P53 group were generally small (Fig. 4c, d). Taken together, these data demonstrate that the recombinant virus rNDV-P53 have a very significant effect in tumor inhibition comparing with rNDV.

In this study, the survival time course of the tumorbearing mice was also recorded. Over the 120 days (Fig. 4e), remaining $7 / 8$ mice in the rNDV group developed significant size and needed to be sacrificed. While only $2 / 8$ mice in the rNDV-P53 group developed tumors that required to be sacrificed. The remaining mice in each group either completely cleared or had persistent pigmented focus that did not change in size. The overall survival of the mice in the long-term study was $0 / 8$ for control group, 6/8 for rNDV-P53 group and $1 / 8$ for rNDV group.

As shown in Fig. 5a, H\&E staining tumor sections show tumors derived from rNDV-P53 demonstrated a stronger suppression to cellar viability than rNDV and PBS group. Then we evaluated the cytotoxic effect of the recombinant virus rNDV-P53 on tumor issue by TUNEL assay. Compared with a control group, we found a significant increase in the apoptosis rate of the rNDV-P53 group (Fig. 5b). These data indicate that rNDV-P53 induces apoptosis, consequently inhibiting the growth of tumor tissue.

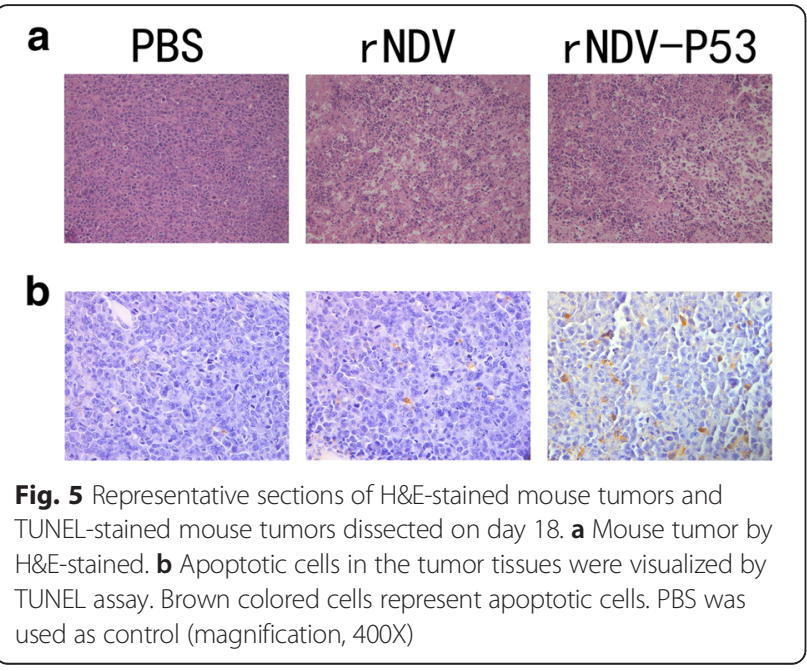

In order to assess the safety and reliability of the recombinant virus administration in vivo, a toxicity study was performed in mice. After ten injections, the blood of mice injected with PBS and rNDV-P53 was collected. Serum chemistries revealed insignificant changes in BUN, creatinine levels, AST and ALT (Fig. 6). According to the results, we concluded the systemic effects of the rNDV-P53 treatment at mice were similar to the PBS treatment. Briefly, the recombinant virus is safe.

\section{Discussion}

Newcastle disease virus (NDV) has a number of advantages as an attractive candidate for viral vector for cancer therapy. First of all, NDV can selectively replicate in tumor cells and cause lysis of cancerous cells while sparing normal cells [30-32]. Secondly, NDV mediates antitumor effects by inducing apoptosis in cancer cells. Multiple subsequent studies confirmed that the apoptosis plays a dominant role in NDV-induced cell death, and the apoptosis inducing effect does not depend on the presence of functional P53 protein in the infected tumor cells [33-36]. Lastly, NDV is a bird virus without pathogenicity in humans, in extreme cases of virus infections, humans only have mild conjunctivitis, laryngitis and flu-like symptoms. In addition, as a viral vector, NDV does not integrate into the human genome [37-40]. These facts contribute to its safety.

The tumor suppressor P53 is a stress-activate transcription factor that regulates several genes with a broad range of functions including DNA repair, apoptosis, cell cycle arrest, metabolism and senescence [20]. In approximately $50 \%$ of human tumors, P53 gene is mutated and losses its function [41]. It is the reason why P53 has emerged as a promising target of gene therapy techniques [42]. P53 gene therapy is aim to prevent tumor formation or lead to tumor regression by restoring the activity of P53. So a strategy has been provided to eliminate tumor cells and induce apoptosis by reintroducing wild-type P53 into tumor cells. In the early 1990s, scientists delivered P53 into tumor cells to induce apoptosis and growth inhibition [43]. Later studies demonstrated the effectiveness of P53 gene therapy, for instance the recombinant adenovirus expressing human P53 achieved good curative effect on malignant liver cancer, gastric cancer, bladder cancer and cervical cancer [44-47].

Due to the superiority of Newcastle disease virus as a vector for cancer therapy and the effectiveness of P53 gene therapy, we constructed the recombinant virus rNDV-P53 using reverse genetics. As we expected, the kinetics and magnitude of replication of the recombinant NDV didn't have a significant change, the P53 expression increased in the tumor cells infected with rNDVP53. In addition, we have shown that compared with the vehicle virus, rNDV-P53 significantly inhibited HepG2 
ALT

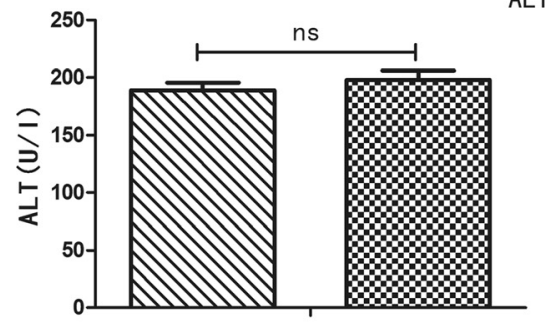

BUN

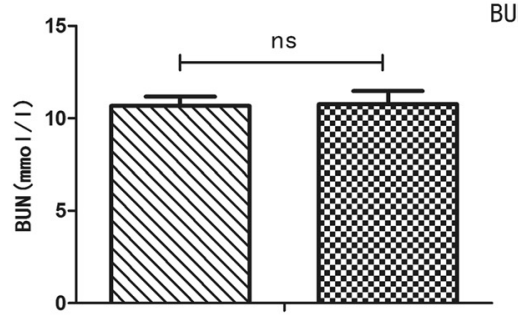

AST
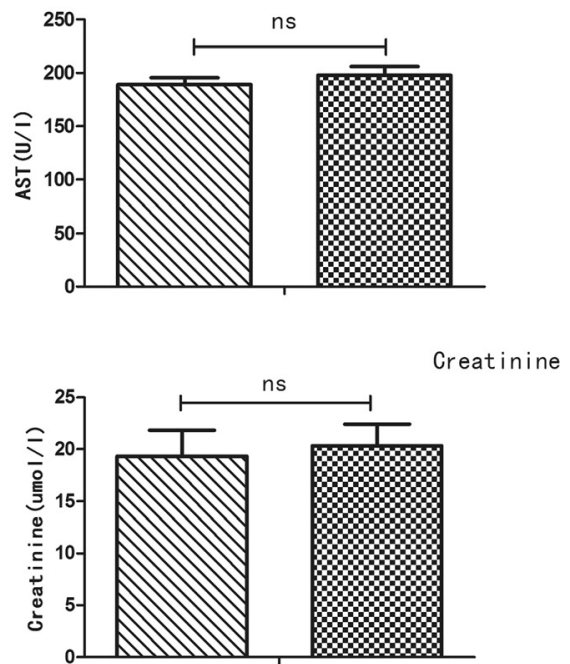

rNDV-P53

Fig. 6 The serum biochemistry data of the experimental mice after injection of rNDV-P53. After ten injections, the blood of mice was drawn for measurement of serum chemistries. Values are expressed as mean \pm SD. ALT, alanine aminotransferase; AST, aspartate transaminase; BUN, blood urea nitrogen; PBS, phosphate-buffered saline; ns, no significant

cells growth. We hypothesized that the ability of rNDVP53 in inducing cell death was enhanced by the inserted gene P53. To confirm this conjecture, we tested this hypothesis in cancer cell lines HepG2. When the cells were infected with virus of 10 MOI for $24 \mathrm{~h}$, the early apoptosis rate induced by rNDV-P53 was $21.1 \%$ and the vehicle virus was $10.4 \%$. Apoptosis analysis showed that rNDV-P53 strengthens pro-apoptotic activity of virus. It is consistent with the previously report that restoring wild-type P53 activity leads to apoptosis in cancer cells [48] Previous investigation suggests that the apoptosis induced by P53 is accompanied by the decrease of mitochondrial membrane potential [49]. Using the fluorescent dye JC-1, we observed a further loss of membrane potential causing by the rNDV-P53 compared with its parental stain (Fig. 3c). Based on the encouraging data in vitro, we investigated the therapeutic efficacy of rNDVP53 in H22-bearing mice. At the end of the experiment, the tumor weights and volumes significantly decreased in mice treated with rNDV-P53 compared with those treated with rNDV or PBS. The average tumor volume in rNDV-P53 group was $666 \mathrm{~mm}^{3}$ verses over $2000 \mathrm{~mm}^{3}$ in rNDV or PBS group. The average tumor weight in rNDV-P53 group was $0.64 \pm 0.24 \mathrm{~g}$ verses 3.52 $\pm 0.33 \mathrm{~g}$ in rNDV group and $6.14 \pm 0.81 \mathrm{~g}$ in PBS group, respectively. These results indicate that rNDV-P53 can effectively inhibit tumor growth. In addition, TUNEL experimental results showed that the rNDV-P53 has a stronger ability to induce apoptosis in tumor tissue than rNDV. rNDV-P53 significantly increased the survival rate of mouse liver cancer model. At the end of the 120 days survival experiment, the overall survival of the untreated model mice was $0 / 8$ verse $6 / 8$ for the rNDVP53 group and 1/8 for the rNDV group. In order to assess potential toxicities associated with recombinant virus in vivo, a toxicity study was performed. AST and ALT are the important indexes in evaluating liver function, the blood urea nitrogen and creatinine are the important indexes in evaluating renal function. We have detected the BUN, creatinine, AST, and ALT. Results showed that these parameters were in the normal range, indicating that rNDV-P53 is relatively safe for cancer therapy.

\section{Conclusion}

In conclusion, we demonstrate a promising oncolytic agent rNDV-P53 consisting of an ideal vector and a suppressor gene P53. Our studies demonstrated that rNDV-P53 is efficacious in suppressing hepatocellular carcinoma model and has the potency to cure the mice completely. Thereby we offered a promising antitumor agent for hepatoma.

\section{Abbreviations}

ALT, alanine aminotransferase; AST, aspartate transaminase; BUN, blood urea nitrogen; DMEM, Dulbecco's modified Eagle medium; FBS, fetal bovine serum;GE, gene end; GS, gene start; H\&E, haematoxylin/eosin; JC-1, 5, 5', 6, 6'-tetrachloro-1, 1', 3, 3'-tetra-ethylbenzimidazolylcarbocyanine iodide; MOl, multiplicity of infection; MTT, microculture tetrazolium; NDV, Newcastle disease virus; PBS, phosphate buffer saline; PFU, plaque forming unit; $T_{C I D}$, tissue culture infectious dose 50; TUNEL assay, terminal deosynucleotidy transferase-mediated dUTP nick and labeling assay 


\section{Acknowledgements}

Not applicable.

\section{Funding}

This study was supported by grants from the National Natural Science Fund biologic science base improve program of research training and capacity (J1210069/J0124), the Heilongjiang province project applies technology research and development (GC13C105), and the Heilongjiang province project applies technology research and development (GC13C104).

\section{Availability of data and materials}

Not applicable.

\section{Authors' contributions}

This study was conceived and designed by $Y A, L T, J H, G R$ and DL. YA, LT, JH and $R C$ analyzed the data and wrote the manuscript. $Y A, T L, J H, R C, Y W, Y B$, $X G, Q Z$ and $C L$ performed the experiments. JY, GR and DL contributed reagents/materials/analysis tools and animal surgery. All authors have read and approved the final manuscript

\section{Competing interests}

The authors declare that they have no competing interests.

\section{Consent for publication}

Not applicable.

\section{Ethics approval and consent to participate}

All procedures involving animals followed the guidelines issued by Nationa Institute of Health and the Institutional Animal Care and Use Committee of Northeast Agriculture University (approval number: SCXK-2011-0004).

\section{Author details}

'Biopharmaceutical Lab, College of Life Science, Northeast Agriculture University, Mucai Street 59, Xiangfang district, Harbin, People's Republic of China. ${ }^{2}$ Key Laboratory of Agricultural Biological Functional Gene, Northeast Agricultural University, Harbin 150030, China.

\section{Received: 14 December 2015 Accepted: 13 July 2016}

\section{Published online: 28 July 2016}

\section{References}

1. Mayo MA. A summary of taxonomic changes recently approved by ICTV. Arch Virol. 2002;147:1655-63.

2. Nemunaitis J. Live viruses in cancer treatment. Oncology (Williston Park) 2002;16:1483-92.

3. Umansky V, Shatrov VA, Lehmann V, Schirrmacher V. Induction of NO synthesis in macrophages by Newcastle disease virus is associated with activation of nuclear factor-kapp B. Int Immunol. 1996:8:491-8.

4. Vigil A, Martinez O, Chua MA, Garcia-Sastre A. Recombinant Newcastle disease virus as a vaccine vector for cancer therapy. Mol Ther. 2008; 16:1883-90

5. Lorence RM, Pecora AL, Major PP, Hotte SJ, Laurie SA, Roberts MS, et al. Overview of phase studies of intravenous administration of PV701, an oncolytic virus. Curr Opin Mol Ther. 2003;5:618-24.

6. Zamarin D, Vigil A, Kelly K, Garcia-Sastre A, Fong Y. Genetically engineered Newcastle disease virus for malignant melanoma therapy. Gene Ther. 2009:16:796-804.

7. Lorence RM, Rood PA, Kelley KW. Newcastle disease virus as an antineoplastic agent: induction of tumor necrosis factoralpha and augmentation of its cytotoxicity. J Natl Cancer Inst. 1988;80:1305-12.

8. Lam HY, Yeap SK, Rasoli M, Omar AR, Yusoff K, Suraini AA, et al. Safety and clinical usage of newcastle disease virus in cancer therapy. J Biomed Biotechnol. 2011;13:1-13

9. Vigil A, Park MS, Martinez O, Chua MA, Xiao S, Cros JF, et al. Use of reverse genetics to enhance the oncolytic properties of Newcastle disease virus. Cancer Res. 2007;67:8285-92

10. Livingstone LR, White A, Sprouse J, Livanos E, Jacks T, Tlsty TD. Altered cell cycle arrest and gene amplification potential accompany loss of wild-type p53. Cell. 1992;70:923-35.
11. Zhao H, Janke M, Fournier P, Schirrmacher V. Recombinant Newcastle disease virus expressing human interleukin-2 serves as a potential candidate for tumor therapy. Virus Res. 2008;136:75-80.

12. Elankumaran S, Rockemann D, Samal SK. Newcastle disease virus exerts oncolysis by both intrinsic and extrinsic caspase-dependent pathways of cell death. J Virol. 2006:80:7522-34.

13. Dortmans JC, Koch G, Rottier PJ, Peeters BP. Virulence of Newcastle disease virus: what is known so far. Vet Res. 2011:42:347-67.

14. Fabian Z, Csatary CM, Szeberenyi J, Csatary L. p53-indenpendent endoplasmic reticulum stress-mediated cytotoxicity of a Newcastle disease virus strain in tumor cell lines. J Virol. 2007:81:2817-30.

15. Fabian Z, Vecsernyes M, Pap M, Szeberenyi J. The effects of a mutant p53 protein on the proliferation and differentiation of $\mathrm{PC} 12$ rat phaeochromocytoma cells. J Cell Biochem. 2006;99:1431-41.

16. Clarke AR, Purdie CA, Harrison DJ, Morris RG, Bird CC, Hooper ML, et al. Thymocyte apoptosis induced by p53-dependent and independent pathways. Nature. 1993:362:849-52.

17. Reichard KW, Lorence RM, Cascino CJ, Peeples ME, Walter RJ, Fernando MB, et al. Newcastle disease virus selectively kills human tumor cells. J Surg Res. 1992;52:448-53.

18. Mihara M, Erster S, Zaika A, Petrenko O, Chittenden T, Pancoska P, et al. p53 has a direct apoptogenic role at the mitochondria. Mol Cell. 2003;11:577-90.

19. Harris CC, Hollstein M. Clinical implications of the p53 tumor-suppressor gene. N Engl J Med. 1993;329:1318-27.

20. Diller L, Kassel J, Nelson CE, Gryka MA, Litwak G, Gebhardt M, et al. p53 functions as a cell cyle control protein in osteosarcomas. Mol Cell Biol. 1990;10:5772-81.

21. Desagher S, Osen-Sand A, Nichols A, Eskes R, Montessuit S, Lauper S, Maundrell K, Antonsson B, Martinou JC. Bid-induced conformational change of Bax is responsible for mitochondrial cytochrome $\mathrm{c}$ release during apoptosis. J Cell Biol. 1999;144:891-901.

22. Chipuk JE, Kuwana T, Bouchier-Hayes L, Droin NM, Newmeyer DD, Schuler $M$, et al. Direct activation of Bax by p53 mediates mitochondrial membrane permeabilization and apoptosis. Science. 2004:303:1010-4

23. Hainaut $P$, Hollstein M. p53 and human cancer: the first ten thousand mutations. Adv Cancer Res. 2000;77:81-137.

24. Vogelstein B, Lane D, Levine AJ. Surfing the p53 network. Nature. 2000:408:307-10.

25. Ventura A, Kirsch DG, McLaughlin ME, Tuveson DA, Grimm J, Lintault L, et al. Restoration of p53 function leads to tumor regression in vivo. Nature. 2007:445:661-5.

26. Cheok CF, Verma CS, Baselga J, Lane DP. Translating p53 into the clinic. Nat Rev Clin Oncol. 2011:8:25-37.

27. Roxburgh P, Hock AK, Dickens MP, Mezna M, Fisher PM, Vousden KH. Small molecules that bind the Mdm2 RING stabilize and activate p53. Carcinogenesis. 2012;33:791-8.

28. Nielsen LL, Dell J, Maxwell E, Armstrong L, Maneval D, Catino JJ. Efficacy of p53 adenovirus-mediated gene therapy against human breast cancer xenografts. Cancer Gene Ther. 1997:4:129-38.

29. Clayman GL, Frank DK, Bruso PA, Goepfert H. Adenovirus-mediated wild-type p53 gene transfer as a surgical adjuvant in advanced head and neck cancers. Clin Cancer Res. 1999:5:1715-22.

30. Nelson NJ. Scientific interest in Newcastle disease virus is reviving. J Nat Cancer Inst. 1999;91:1708-10

31. Pecora AL, Rizvi N, Cohen Gl, Meropol NJ, Sterman D, Marshall الـ, et al. Phase I trial of intravenous administration of PV701, an oncolytic virus, in patients with advanced solid cancers. J Clin Oncol. 2002:20: 2251-66.

32. Krishnamurthy S, Takimoto T, Scroggs RA, Portner A. Differentially regulated interferon response determines the outcome of newcastle disease virus infection in normal and tumor cell lines. J Virol. 2006;80: 5145-55.

33. Ravindra PV, Tiwari AK, Ratta B, Bais MV, Chaturvedi U, Palia SK, et al. Time course of Newcastle disease virus-induced apoptotic pathways. Virus Res. 2009;144:350-4

34. Fábián Z, Vecsernyes M, Pap M, Szeberenyi J. The effects of a mutant p53 protein on the proliferation and differentiation of PC12 rat phaeochromocytoma cells. J Cell Biochem. 2006:99:1431-41.

35. Ravindra PV, Tiwari AK, Ratta B, Chaturvedi U, Palia SK, Chauhan RS. Newcastle disease virus-induced cytopathic effect in infected cells is caused by apoptosis. Virus Res. 2009;141:13-20. 
36. Mansour M, Palese P, Zamarin D. Oncolytic specifiity of Newcastle disease virus is mediated by selectivity for apoptosis-resistant cells. J Virol. 2011;85:6015-23.

37. Charan S, Mahajan VM, Agarwal LP. Newcastle disease virus antibodies in human sera. Indian J Med Res. 1981;73:303-7.

38. DiNapoli JM, Kotelkin A, Yang L, Elankumaran S, Murphy BR, Samal SK, et al. Newcastle disease virus, a host range-restricted virus, as a vaccine vector for intranasal immunization against emerging pathogens. Proc Natl Acad Sci U S A. 2007;104:9788-93.

39. Schirrmacher V, Haas C, Bonifer R, Ahlert T, Gerhards R, Ertel C. Human tumor cell modifiation by virus infection: an effiient and safe way to produce cancer vaccine with pleiotropic immune stimulatory properties when using Newcastle disease virus. Gene Ther. 1999;6:63-73.

40. Liang W, Wang H, Sun TM, Yao WQ, Chen LL, Jin Y, et al. Application of autologous tumor cell vaccine and NDV vaccine in treatment of tumors of digestive tract. World J Gastroenterol. 2003;9:495-8.

41. Soussi T, Dehouche K, Beroud C. p53 website and analysis of p53 gene mutations in human cancer: forging a link between epidemiology and carcinogenesis. Hum Mutat. 2000;15:105-13.

42. Rosenfeld MR, Meneses P, Dalmau J, Drobnjak M, Cordon-Cardo C, Kaplitt MG. Gene transfer of wild-type $p 53$ results in restoration of tumor-suppressor function in a medulloblastoma cell line. Neurology. 1995:45:1533-9.

43. Roth JA, Nguyen D, Lawrence DD, Kemp BL, Carrasco CH, Ferson DZ, et al. Retrovirus-mediated wild-type p53 gene transfer to tumors of patients with lung cancer. Nat Med. 1996:2:985-91.

44. Harris MP, Sutjipto S, Wills KN, Hancock W, Cornell D, Johnson DE, et al. Adenovirus-mediated p53 gene transfer inhibits growth of human tumor cells expressing mutant p53 protein. Cancer Gene Ther. 1996;3:121-30.

45. He X, Liu J, Yang C, Su C, Zhou C, Zhang Q, et al. 5/35 Fiber-Modified Conditionally Replicative Adenovirus Armed with p53 Shows Increased Tumor-Suppressing Capacity to Breast Cancer Cells. Hum Gene Ther. 2011 22:283-92.

46. Shi J, Zheng D. An update on gene therapy in China. Curr Opin Mol Ther. 2009:11:547-53.

47. Yang ZX, Wang D, Wang G, Zhang QH, Liu JM, Peng P, et al. Clinical study of recombinant adenovirus-p53 combined with fractionated stereotactic radiotherapy for hepatocellular carcinoma. J Cancer Res Clin Oncol. 2010; 136:625-30.

48. Joerger AC, Ang HC, Fersht AR. Structural basis for understanding oncogenic p53 mutations and designing rescue drugs. Proc Natl Acad Sci U S A. 2006:103:15056-61.

49. Moll UM, Zaika A. Nuclear and mitochondrial apoptotic pathways of p53. FEBS Lett. 2001:493:65-9.

\section{Submit your next manuscript to BioMed Central and we will help you at every step:}

- We accept pre-submission inquiries

- Our selector tool helps you to find the most relevant journal

- We provide round the clock customer support

- Convenient online submission

- Thorough peer review

- Inclusion in PubMed and all major indexing services

- Maximum visibility for your research

Submit your manuscript at www.biomedcentral.com/submit

Biomed Central 